\title{
EL ORIGEN DEL SÍMBOLO DE LA MEDICINA
}

\section{The origin of the symbol of medicine}

\section{*Eduardo José Cartagena Mendoza , **Lourdes Azucena Andrade Mancias.}

\section{RESUMEN}

El simbolismo constituye una de las formas de lenguaje más arcaicas del pensamiento humano. Un símbolo es la expresión artística de una idea en común; y en la medicina, como en otras ciencias, el simbolismo forma parte del lenguaje para la comunicación efectiva. Actualmente, existen dos representaciones del símbolo de la medicina que se han confundido a través del tiempo por una malinterpretación de más o menos un siglo. La intención de este artículo es aclarar la confusión que existe sobre el origen real del símbolo de la medicina. Un total de 137 publicaciones relacionadas fueron encontradas. En la literatura de nuestro país unicamente se encontró una publicación que data de 1947. Las evidencias históricas demuestran que el símbolo representativo de la medicina es la vara de Asclepio-Esculapio y no el caduceo de HermesMercurio que es en realidad el símbolo de los comerciantes.

\section{PALABRAS CLAVE}

Emblemas e insignias, historia, medicina.

\section{ABSTRACT \\ The symbolism is one of the most archaic forms of language in human thought. A symbol is the artistic expression of an idea in common; and in medicine, as in other sciences, symbolism is part of the language for effective communication. Currently, there are two representative symbols of medicine that have been confused over a century by a misinterpretation. The intent of this article is to clear up confusion about the true \\ *Estudiante de Pregrado de Medicina de la Escuela Universitaria de las Ciencias de la Salud de la Universidad Nacional Autónoma de Hondu- ras en el Valle de Sula. \\ **Especialista en Anestesiología / Docente de la Escuela Universitaria de las Ciencias de la Salud de la Universidad Nacional Autónoma de Honduras en el Valle de Sula. \\ Dirigir correspondencia a: eddocartagena@gmail.com / \\ landrademancias@gmail.com \\ Recibido: 09 de febrero 2016, \\ Aprobado: 23 de junio 2016}

origin of the symbol of medicine. A total of 137 related publications were found. In the literature of our country only 1 publication dating back to 1947 was found. The historical evidence shows that the representative symbol of medicine is the rod of Asclepius-Aesculapius and not the caduceus of Hermes-Mercury which is the symbol of traders.

\section{KEYWORDS}

Emblems and insignia, history, medicine.

\section{INTRODUCCIÓN}

Un símbolo (del lat. symbŏlus, y este del gr. $\sigma u ́ \mu ß o \lambda$ os sýmbolos) es un elemento u objeto que por su convención o asociación, se considera representativo de una entidad, de una idea, de una cierta condición, etc. ${ }^{(1)}$ Por ejemplo, la paloma es el símbolo de la paz. No debe confundirse con emblema, definido como una cosa que es representación simbólica de otra y al pie de la cual se escribe algún verso o lema que declara el concepto o moralidad que encierra, ${ }^{(2)}$ por ejemplo, el emblema de la universidad; tampoco debe confundirse con logotipo (logo), que es un grupo de letras, abreviaturas, cifras, etc; fundidas en un solo bloque para facilitar la composición tipográfica, ${ }^{(3)}$ por ejemplo, el logotipo de la Coca-Cola.

El simbolismo constituye una de las formas de lenguaje más arcaicas del pensamiento humano. ${ }^{(4)}$ A pesar del desarrollo de la escritura en las diferentes civilizaciones, no toda la población tuvo acceso a instruirse, aprender a leer y a escribir, por lo que los símbolos continuaron desempeñando un papel preponderante en la información de las sociedades. ${ }^{(5)}$ Los símbolos en medicina han sido utilizados para representar distintas ideas y conceptos. Así tenemos por ejemplo, el símbolo "Rx", una abreviatura del vocablo latino "Recipere", que significaba "tomar" o "toma esto". (6) 
La conformación del símbolo médico ha cambiado a través de las distintas épocas según las costumbres, ideas y creencias religiosas de los pueblos; fue variando a medida que evolucionaron las civilizaciones, las cuales fueron asignándole caracteres propios de cada una, por lo que el símbolo resulta del producto de una larga transformación histórica. ${ }^{(5)}$ En la actualidad dos símbolos se usan como representantes de la medicina: el caduceo de Hermes y la vara de Asclepio, sin embargo, uno de ellos ha sido malinterpretado por la comunidad médica desde hace algo más de un siglo. Las causas de este error mucho tienen que ver con erradas interpretaciones y descuidadas traducciones que con el paso del tiempo han eludido la precisión de una adecuada significación. ${ }^{(7)}$ La intención de esta revisión es aclarar el porqué de la confusión y motivar a corregir el error.

\section{DESARROLLO}

\section{El caduceo de Hermes.}

El diccionario de la Real Academia Española (RAE) define caduceo (del lat. caducěum, y este el gr. кпрúkદıov kērýkeion literalmente 'del heraldo') como la vara delgada, lisa y cilíndrica, rodeada de dos culebras, atributo del dios romano Mercurio y empleada hoy como símbolo del comercio (Figura No. 1.) $^{(8)}$ Los romanos sustituyeron al dios griego Hermes por su equivalente Mercurio. Es por eso que se habla del caduceo de Mercurio. Hermes-Mercurio era el mensajero de los dioses, conductor de los muertos, patrón de los viajeros y protector de los comerciantes y ladrones. ${ }^{(9)}$

Hermes fue hijo del dios Zeus (dios de los dioses, llamado este último Júpiter en la mitología romana) y hermano de Apolo.

Figura No. 1: Caduceo de Hermes-Mercurio.

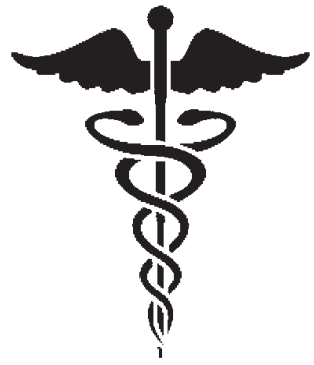

Fuente: Continuing Education Inc. 2016. ${ }^{(10)}$
Era representado como el más joven de los dioses con un casco y sandalias con alas y una vara con 2 serpientes a su alrededor; el caduceo. ${ }^{(9)}$ Este caduceo lo obtuvo luego de haber robado parte de los ganados vigilados por Apolo. (11) Cuando Apolo descubrió el robo, llevó a Hermes ante Zeus, quien lo obligó a regresar el ganado. Sin embargo, Apolo encantado por el sonido de la lira que Hermes había fabricado, la acepto como obsequio de disculpa y este le dio a cambio, la ganadería y el caduceo. Zeus, sorprendido por la vivacidad y la inteligencia de su hijo, lo convirtió en su mensajero y lo puso al servicio de Hades (dios del inframundo y llamado Plutón en la mitología Romana).

Una de las tareas de Hermes era llevar a los muertos al reino de Hades. Este probablemente sea el origen de la costumbre en la antigüedad, de que los hombres que buscaban a los heridos y los muertos en el campo de batalla llevaran el caduceo, similar a la bandera blanca o la bandera de la Cruz Roja en los conflictos recientes. De ahí pudo surgir el hecho de que el caduceo sea malinterpretado como el símbolo de la atención de salud de algunas fuerzas armadas, incluidos los EE.UU. (12)

Otra versión según cuenta Ovidio (43 a.C- 17 d.C.) es que Hermes encontró en el Monte Citerón a dos serpientes que se peleaban, él arrojó en medio de ellas su vara para separarlas y vio cómo, sin hacerse daño, se enroscaron y se entrelazaron alrededor de ella, de forma tal que con la parte más alta de sus cuerpos formaron un arco, quedando sus cabezas frente a frente sin señal de enemistad. Probablemente, de este mito que muestra el complemento de los opuestos, nació el símbolo. Según Schneider, las dos $S$ formadas por las serpientes corresponden a enfermedad y convalecencia. ${ }^{(4)}$

La vara mágica con las serpientes enrolladas fue un símbolo común de varias deidades antiguas, como el dios Thot en Egipto, Tauut en la Fenicios, y Ningizzida en los Sumerios. Primitivamente, fue una rama de olivo, otras veces de laurel, de cabeza nudosa que se bifurcan en 
dos y se enroscan para juntar sus extremos, variando a través de los tiempos y las culturas, para al final adquirir las serpientes y las alas de Mercurio. Los griegos lo tenían como báculo heráldico, representado por una caña entrelazada con hilos de lana; en la Edad Media fue un bastón cubierto de terciopelo flordelisado y así fue cambiando según la época histórica. ${ }^{(9)}$

\section{La leyenda de Asclepio}

La leyenda de Asclepio, que data de alrededor del año 700 a.C., fue reportado por Hesíodo. ${ }^{(12)}$ Autores de la antigüedad, entre los que destacan Homero y Píndaro, mencionan que Asclepio fue realmente un médico famoso y héroe de guerra, que nació en Trica localizada en la región de Tesalia que hoy se conoce con el nombre de Trikala. ${ }^{(13)}$ Él era el hijo del dios Apolo y la ninfa Coronis. Apolo fue considerado el médico de los dioses del Olimpo y segundo en la lista desde Paeon. ${ }^{(14)}$ Cuenta la leyenda, que Coronis había sido prometida en matrimonio por su padre al primo de Apolo, Isquis, pero ella contemplaba su próxima boda llevando al hijo de Apolo en su vientre. Un cuervo, que en aquel entonces era un ave con plumas blancas, llevó la noticia de la próxima boda a Apolo con lo que montó en cólera, le dio plumas negras al cuervo, quien las porta desde entonces como señal de duelo, y disparó luego sus flechas al novio de Coronis (Isquis) mientras que Artemisa, la hermana de Apolo, mataba a la infeliz Coronis. Cuando Apolo vio que su amante moría, se llenó de compasión por el hijo no nacido, de modo que lo tomó del vientre de Coronis y lo llevó a la cueva del centauro Quirón. Quirón (del

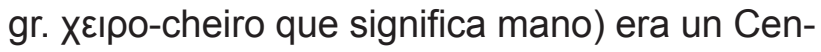
tauro (mitad caballo y mitad hombre) de la guerra muy habilidoso con las manos. No sólo era un diestro cazador y amigo de la música, sino que conocía también las propiedades de las hierbas curativas, las utilizaba y sabía cómo transmitir sus conocimientos a sus discípulos. Quirón fue el preceptor de Hércules, Aquiles y Asclepio. ${ }^{(13)}$ Asclepio aprendió el arte de curar y se convirtió pronto en un médico de renombre en la Grecia. Llegó a ser tan experto en el arte que podía resucitar a los muertos. ${ }^{(12,15,16)}$
Una vez fue a sanar a Glaukos, un enfermo terminal al que le había caído un rayo, y mientras estaba en casa de éste, una serpiente estaba a punto de morder al paciente por lo que la mato con su vara. Sin embargo, luego vino otra con unas hierbas en su boca y se las dio a la serpiente muerta y esta revivió. Asclepio hizo lo mismo con su paciente y el enfermo revivió.

Asclepio era representado como un hombre joven de barba, vistiendo una toga y con la mitad del pecho derecho descubierto sosteniendo un bastón, en el cual se enroscaba una serpiente, en la mano del mismo lado, su esposa Epione, se dice que calmaba el dolor. Sus hijas fueron Higia y Panacea; la primera fue conocida como la deidad de la higiene y la profilaxis y Panacea la del tratamiento que todo lo sanaba. Sus hijos, Macaón y Podalirio, fueron grandes cirujanos durante la guerra de Troya según menciona Homero en la líada, mientras que Telésforo, quien le acompañaba siempre, significaba la convalecencia. ${ }^{(13)}$ La muerte de Asclepio sucede después de que Hades le reclama a Zeus que Asclepio no dejaba ir a los muertos al inframundo, debido a que los revivía, por lo que Zeus decide matarlo con un rayo para mantener el orden natural de las cosas. En otra versión, Asclepio fue asesinado por las flechas de su propio padre. ${ }^{(12)}$

La deidad de Asclepio (mitad dios y mitad humano) no se instituyo hasta un poco más tarde. El culto a Asclepio tuvo su origen en Tesalia. ${ }^{(13)}$ No se sabe de cierto si en tiempo de Homero, se le consideraba ya como dios o sólo era un médico. En el 420 a.C., una plaga devastó la ciudad de Atenas la cual, por intercesión de Asclepio (ya muerto), fue detenida y desde entonces fue proclamado dios del Olimpo. Platón lo menciona en las últimas palabras de Sócrates: "Crito, le debemos un gallo a Asclepio, págaselo por favor y no lo descuides".

En los tiempos de Hipócrates se le menciona tal y como el mismo lo expresa en su juramento: "Juro por Apolo médico y Asclepio, Hegéia y Panacea..."(12) Se piensa que Hipócrates de Cos fue descendencia directa de Asclepio pero no 
hay evidencia concreta que lo demuestre. La fama de Asclepio siguió creciendo por todo el oeste del Mediterráneo y Asia menor donde se construyeron templos en los cuales se curaban a los enfermos con serpientes no venenosas de la especie Elaphis Aesculapii ${ }^{(14)}$ y el hábito de la incubación. ${ }^{(13)}$ Este último consistía en que durante el sueño, en un lugar especial del templo llamado "dormitorio", Asclepio se les aparecía a los enfermos y los sanaba con el toque de la vara. Además de la incubación se practicaba en los templos la dieta, el ejercicio, la higiene, la hidroterapia y la cirugía. ${ }^{(15)}$

Estos templos (Asclepiones) se ubicaron en varias ciudades (más de 300 santuarios), pero el más importante era el Epidauro. A los sanadores que pertenecian a los Asclepiones se les llamaba Asclepíades. ${ }^{(13)}$ En el 293 a.C., una peste ataco Roma donde los doctores de esa ciudad no pudieron curarla y acudieron al oráculo de Delphi en búsqueda de ayuda, éste les instruyo que siguieran los consejos de Asclepio, los romanos entonces llevaron a la deidad, representada como una serpiente enrollada en una vara, a su ciudad y curo la peste. Incluso Galeno recurrió a él para sanar al emperador Marco Aurelio. ${ }^{(9,15)}$ Los romanos sustituyeron el nombre de Asclepio por el de Esculapio y por eso hoy se le conoce también al bastón como vara de Esculapio (pero no caduceo).

\section{La vara de Asclepio}

La vara (bastón o báculo) de Asclepio se diferencia del caduceo de Hermes en que solo es una vara con una serpiente enrollada (Ver figura No. 2). La vara siempre ha simbolizado autoridad y poder, ${ }^{(16)}$ y se dice que las personas encargadas de sanar en la antigua Grecia la llevaban consigo, junto a las medicinas e instrumentos, para ayudarse a caminar debido a que no tenían establecido un lugar para ejercer el arte y recorrían largos caminos para encontrar trabajo. La vara ha sido asociada también al arte de sanar porque un método para retirar filarias y nematodos como Dracunculus medinensis era el de introducir una vara subcutáneamente y enroscar el gusano sobre ella para extraerlo. ${ }^{(9)}$
La vara en su punto más alto tiene un botón que representa, según varios autores, los conflictos de la ciencia. El material de la vara se ha dicho que es de roble o ciprés (conocido antiguamente como árbol de la vida). La serpiente que se observa en el bastón es de la familia Colubridae y de la especie Elaphe longissima. ${ }^{(17,18)}$ En la historia, se ha visto que la serpiente ha sido venerada y respetada por muchas culturas. ${ }^{(12)}$ Su papel en todas estas lo podemos leer en el trabajo de Frederick Stenn. ${ }^{(19)}$

\section{Figura No. 2: Vara de Asclepio-Esculapio}

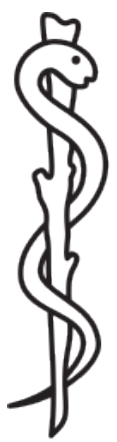

Fuente: Prates Paulo R. Do bastão de Esculápio ao caduceu de Mercúrio. Arq. Bras. Cardiol. 2002. ${ }^{(20)}$

El indicio más antiguo es en Babilonia, donde el dios de la fertilidad Ningizza es representado por dos serpientes enrolladas sobre una vara acompañadas de dos grifos. ${ }^{(9)}$ Esta representación muestra que la vara con dos serpientes es más antiguo que el bastón de Asclepio. En Mesopotamia, la serpiente Sachan, símbolo de salud, recibía culto como figura representativa del demonio. Según la literatura mesopotámica existían siete demonios, terribles especialistas en atacar una determinada parte del cuerpo. Cuando no se adoraba a la serpiente, se le hacía participar en las libaciones, y se hacían sacrificios en ofrenda a ella; de esta manera, según sus creencias, si la tenían satisfecha y lograban sus deseos, esta les retribuía en salud. ${ }^{(21)}$ En los orígenes de la civilización griega, el culto a la serpiente era desconocido, siendo probable que fuera importado de Egipto o de Asiria. ${ }^{(13)}$ Los Faraones egipcios la utilizaban en sus frentes (Ureus) ya que creían que escupía fuego y veneno ante amenazas. ${ }^{(11)}$

Para las culturas precolombinas, como la azteca 
y maya, la serpiente emplumada, Quetzalcóatl y Kukulcan respectivamente, tenía significado de deidad. Los indios de América del Norte rindieron tributo a la serpiente de cascabel; los indios del Amazonas a la anaconda, los budistas a la cobra y los babilónicos a la pitón. ${ }^{(4)}$ En la India y la cultura Hindú se tienen datos de este tipo ${ }^{(9)}$ así como también en la cultura China con su serpiente voladora, el Dragón. Hay evidencia que sustenta que este símbolo existía ya en China a pesar de no existir comunicación entre oriente y occidente para esa época, por lo que el simbolismo de la serpiente representa un pensamiento común de la humanidad. ${ }^{(22)}$ Incluso la comunidad judío-cristiana narra en el libro del Génesis la figura de una serpiente en el jardín del Edén ${ }^{(23)}$ así como también en la historia de Moisés cuando uso una serpiente para sanar al pueblo por la desobediencia ${ }^{(24)}$ o al presentarse al Faraón. ${ }^{(25)}$ Por tanto, es un hecho que la serpiente es el símbolo universal de lo sobrenatural en la prehistoria y en el ámbito de la mitología griega simboliza la prudencia, previsión, sabiduría, agilidad, destreza, salud, longevidad, convalecencia y rejuvenecimiento; por lo que ha representado para la humanidad los poderes mágicos de sanación y curación que se han atribuido a todas las divinidades médica. ${ }^{(11,13,15)}$

La capacidad de la serpiente de mudar su piel indicaba longevidad e inmortalidad. La habilidad de cambiar de un estado de letargo a otro de rápida actividad representa el poder de pasar de un estado de convalecencia a otro de curación. ${ }^{(16)}$ Además, hay que agregar la ironía de que, a pesar de poder envenenar, la serpiente tenía también la cura de su propio veneno, por lo que las propiedades sanadoras atribuidas a esta eran aún más especiales. A pesar de lo anterior, el papel de la serpiente como símbolo de sanación aun es discutido. ${ }^{(21)}$

\section{Malinterpretación}

Para el siglo VII, Hermes-Mercurio se asociaba con la alquimia y los alquimistas eran llamados Hermeticistas. Fue en el siglo XVI cuando ocurren las primeras confusiones entre el caduceo y la vara de Asclepio. De hecho, el principal motivo de confusión en los tiempos modernos fue cuando Hieronymus Froben (1501-1563), hijo de Johannes Froben, imprimió algunos libros médicos con el símbolo del caduceo, entre los cuales se encontraban una edición en griego de los trabajos de Hipócrates (1538). El caduceo fue el símbolo de su tipografía y, como tal, fue estampado en la portada del libro. Sir William Butts (1486-1545), médico de Enrique VIII, fue autorizado para usar el caduceo como distintivo profesional y posteriormente se incluyó en los emblemas del Royal College of Physicians de Londres. ${ }^{(4)}$

Además, en ese mismo siglo la casa editorial John Churchill imprimió varios escritos de medicina usando como distintivo el caduceo. ${ }^{(26,7)}$ Luego para el siglo XVII, Hermes no solo era asociado con la alquimia sino también con la astrología, magia, medicina y las ciencias ocultas. Otra razón que atribuyen algunos autorespara la confusión es que el caduceo pertenecía a Apolo el cual se lo dio a Mercurio a cambio de la lira. ${ }^{(12)}$

Sin embargo, no fue hasta 1902 cuando la confusión se generalizó por todo el mundo cuando el Cuerpo Médico de las Fuerzas Armadas de Estados Unidos decidió utilizarlo en sustitución de la Cruz de San Juan. ${ }^{(4)}$ Garrinson, un cirujano del ejército e historiador, fue entrevistado del porqué del cambio y del error, pero no pudo explicarlo. Una carta que envió en 1932 cita: "el caduceo, no como un símbolo médico, sino como un símbolo administrativo, implica el estado neutral, no combatiente del personal en el campo de batalla"; (16) justificando de esta manera el propósito de su uso, a pesar de que se conocía para entonces la vara de Asclepio como símbolo de la medicina y a pesar de que las respuestas no se hicieron esperar por muchos doctores al darse cuenta del error. Sin embargo, el uso de tal símbolo para fines "exclusivamente" de guerra, permitió que con el paso del tiempo se mantuviera el símbolo.

En Corea por ejemplo, la Asociación Coreana de Medicina (KMA por sus siglas en inglés) mantiene el caduceo como símbolo de la medicina 
debido a la influencia de la Armada Estadounidense durante la década de los 40.(11) Otros países como Nicaragua, Ecuador, Brasil, República Dominicana, Honduras, etc; aún usan entre sus instituciones de salud el caduceo como distintivo. $^{(4)}$

El símbolo de la vara de Esculapio (que no debe llamarse caduceo de Esculapio o de medicina) ${ }^{(7)}$ fue adquiriendo modificaciones y la Organización Mundial de la Salud (OMS) lo sustenta desde su fundación en 1947. En 1919 la Asociación Médica de Estados Unidos y en 1956 la Asociación Médica Mundial lo adoptaron como sus símbolos. ${ }^{(12)}$ El símbolo desapareció en el siglo IV, cuando el cristianismo fue declarado religión oficial del imperio Romano y con ello se eliminaron todos los dioses y símbolos paganosde la época.

Fue hasta los tiempos de la Reforma, en el siglo $X V I$, cuando resurgió luego de que muchas imprentas comenzaron a publicarlo en libros de la medicina por toda Europa. En la ciudad de Cos, aún se puede observar en sus muros la representación de la vara de Asclepio como símbolo de la medicina pero nunca la del caduceo de Hermes. ${ }^{(27)}$

Muchas organizaciones y países como Gran Bretaña, Alemania, México, Perú, Bélgica, Filipinas, Cuba, entre otros; ${ }^{(26)}$ usan la vara de Esculapio como símbolo que representa a la medicina. En Cuba sin embargo, se usa una modificación del símbolo, en el que hay un espejo en la parte más alta de la vara (que se cree son uvas estilizadas) y además dos ramas (una de laurel y otra de roble) rodeándolo (Figura No. 3). El racimo de uvas significa el vino que es usado, con fines proféticos y adivinadores en la práctica misteriosa de los taumaturgos, y en la terapéutica, como medicamento preciado, proveniente de la vid, árbol que procura la vida y el amor.

Desde hace un tiempo se acepta el espejo, ya que en Egipto el espejo era mágico y al tomar un narcótico, en este se veían "seres sobrenatura- les". ${ }^{(17,26)}$ Algo interesante, es que el símbolo se ha mantenido a través del tiempo por pura tradición, de manera que, si se quisiera, se pudiera cambiar a uno más acorde con nuestros tiempos.

Figura No. 3: Símbolo del Ministerio de Salud Pública de Cuba.

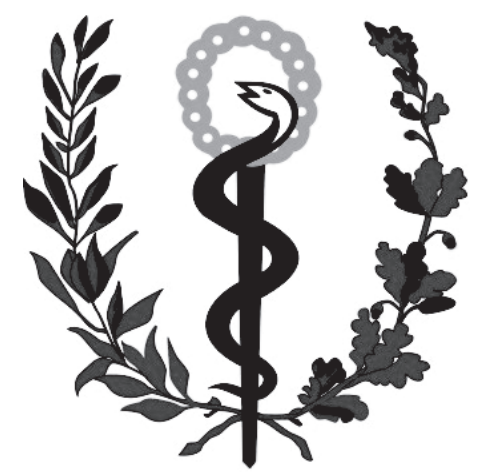

Fuente: Vidal M, Borroto ER, Delgado G. El caduceo de la medicina, símbolo distintivo y emblema oficial de la Salud Pública Cubana. Educ Med Super. 2004. ${ }^{(28)}$

Es decir, a pesar de que no podemos desligar el pasado de nuestra cultura, si se puede, con los avances extraordinarios del conocimiento actual, mejorar lo que no está bien según lo dicte la razón y lo demuestre la evidencia. El Dr. Alvabez ${ }^{(21)}$ cita al respecto: "el mundo médico ha aceptado este simbolismo, por tradición, por la fuerza de la costumbre, pasivamente, sin reflexión ni crítica alguna y sobre todo sin darle importancia alguna. Pero tiene que llegar el día en que el símbolo desaparecerá y se adoptará otro, en consonancia con la razón y con las glorias de la clase médica, cuya influencia en el progreso humano es enorme y de la que han salido numerosos e insignes benefactores de la humanidad".

Algunos autores sugieren por ejemplo, que deberían de ser las flechas las que representarían mejor al gremio, ya que Apolo las utilizaba en sus luchas y representarían la certeza y las numerosas posibilidades de diagnóstico y tratamiento que puede tener un paciente. ${ }^{(29)}$

Nayernouri( ${ }^{(9)}$ en Iran por ejemplo, recomienda el uso de otra deidad más representativa para esa cultura (el ave Simurgh) que la usada por los griegos. 


\section{CONCLUSION}

El símbolo de la medicina debe expresar en nosotros el profundo sentimiento de entrega a nuestros pacientes. Aun cuando la medicina de nuestros tiempos se pueda regir por relaciones de oferta y demanda y aun cuando se tienda a ver al paciente como usuario (en cuyo caso si estaría justificado el uso del caduceo de Hermes-Mer curio), no debemos olvidar el fin supremo de la carrera que es el de curar y no hacer daño. Esta actitud encaja mejor con el significado de la vara de Asclepio que es el verdadero símbolo de la medicina. Se considera por tanto, que el ejercicio hipocrático de la medicina corresponde totalmente con lo que representó Esculapio y el símbolo de este, por ser Hipócrates su seguidor y concebir este ejercicio en el marco de la ética y moral rigurosa que debe caracterizar el desempeño de nuestra profesión.

En la Escuela y Facultad de Medicina de la Universidad Nacional Autónoma de Honduras, seguimos utilizando el caduceo de Hermes-Mercurio como símbolo representativo de la medicina, incluso el Colegio Médico y la Secretaria de Salud de nuestro país lo utilizan erróneamente. Quizás esto se deba a la falta de conocimiento del tema debido a que es algo que no se enseña en las aulas de clase. Esperamos que esta revisión impulse un cambio de concepción en profesionales y estudiantes sobre el origen real del símbolo de la medicina.

\section{BIBLIOGRAFÍA}

1. Diccionario de la lengua española. 23a ed. Madrid: Real Academia Española; 2014. Símbolo. [citado 2016 Ene 20] Disponible en: http://dle.rae.es/?id=Xu q7wTS.

2. Diccionario de la lengua española. 23a ed. Madrid: Real Academia Española; 2014 Emblema. [citado 2016 Ene 20] Disponible en: http://dle.rae.es/?id=Ee1 R68A.

3. Diccionario de la lengua española. 23a ed. Madrid: Real Academia Española; 2014. Logotipo. [citado 2016 Ene 20] Disponible en: http://dle.rae.es/?id=NZg7mG3.

4. Young Pablo, Finn Bárbara C, Bruetman Julio E, Cesaro Gelos Jorge, Trimarchi Hernán. Rod of Asclepius. Symbol of Medicine. Rev. med. Chile [Internet]. 2013 Sep [cited 2016 Jan 20]; 141(9): 1197-1201. Disponible en: http:// www. scielo.cl/scielo. php?script=sci_arttext\&pid=S0034-98872 013000900013\&lng=en. http://dx.doi.org/ 10.4067/S00 34-98872013000900013.

5. Pérez Vázquez Isael Armando, Sánchez Lera Rita María. El bastón de Esculapio: su historia. Rev Hum Med [revista en la Internet]. 2014 Abr [citado 2016 Ene 20]; 14(1): 220-237. Disponible en: http://scielo. sld.cu/scielo.php?script=sci_arttext $\&$ pid= S1727-8120201400010 0014\& $\mathrm{Ing}=\mathrm{es}$.

6. Lain L. O. Buxton. Principios de la redacción de prescripciones médicas (recetas) e instrucciones para el apego del paciente a la prescripción. En: Laurence L. Bruton, editor. Goodman and Gilman: Las bases farmacológicas de la terapéutica clínica. 12a ed. México: Mc Graw-Hill; 2012. p. 1879.

7. Hernández Perera Julio César. Vara, báculo y bastón, pero no caduceo. Rev. cub. Salud pública [Internet]. 2012 Dec [cited 2016 Jan 20]; 38(4): 673-675. Disponible en: http:// www.scielosp.org/ scielo.php?script=sci_ar ttext\&pid=S08 64-34662012000400018\&In $\mathrm{g}=$ en.http://dx.doi.org/10.1590/S0864-34 662012000400018.

8. Diccionario de la lengua española. 23a ed. Madrid: Real Academia Española; 2014. Caduceo. [Citado 2016 Ene 20] Disponible en: http://dle.rae.es/?id=6b JaGcA. 
9. Nayernouri, Touraj. Asclepius, Caduceus, and Simurgh as Medical Symbols Part I. Arch Iran Med [Internet]. 2010 Jan [citado en 2016 Jan 20]; 13 (1): 61-68. Disponible en: http://www.ams.ac.ir/AIM/NEWPUB/10/ 13/1/0015. pdf.

10. Continuing Education.net [internet] Continuing Education, Inc. [actualizado 2016; cited 2016 June 12] Disponible en: http:/ /www.continuingeducation.net/images/logo _Cadaceus.jpg.

11. Shetty, A., Shetty, S., \& Dsouza, O. Medical Symbols in Practice: Myths vs Reality. Journal of Clinical and Diagnostic Research: JCDR [Internet]. 2014 Aug [Citado en 2016 Jan 31]; 8(8): 12-14. Disponible en: http://doi.org/10.7860/JCDR/2014/100 29.4730 .

12. Prates Paulo R. Do bastão de Esculápio ao caduceu de Mercúrio. Arq. Bras. Cardiol. [Internet]. 2002 Oct [cited 2016 Jan 20]; 79( 4): 434-436. Disponible en: http://www. scielo.br/scielo.php?script=sci_arttext\&pid= S0066-782X2002001300014\&Ing=en . http://dx.doi.org/10.1590/S0066-782X200 2001300014.

13. Rillo, A. The Greek origin of caduceum: Esculapius. Colombia Médica [Internet]. 2009 [citado 2016 Jan 20]; 39(4): 384-393. Disponible en: http://colombia medica.univalle.edu.co/index.php/come dica/article/view/620/872.

14. Lips Castro W, Urenda Arias C. A review of the principle mythical gods in ancient Greek medicine. Gac Med Mex [Internet]. 2014 Dec [citado en 2016 Jan 20]; 3(150): 377-85. Disponible en: http:// www.anmm. org.mx/GMM/2014/s3/GMM_150_2014_S3 _377-385.pdf.

15. Kelly ADA. Crash of symbols. Canadian Medical Association Journal [Internet]. 1973 [citado en 2016 Jan 20]; 109(6): 515.
Disponible en: http://www.ncbi.nl m.nih. gov/pmc/articles/PMC1946924/

16. Coston. Tullos O. The proper symbol of medicine. AM. OPHTH. SOC [Internet]. 1970 [Citado en 2016 Jan 20]; 68: 359 363. Disponible en: http://www.ncbi.nlm. nih.gov/pmc/articles/PMC1310384/.

17. Creagh Peña Mabel, González Serrano Delfina. En defensa de la identidad médica cubana. Rev. cub. Salud pública [Internet]. 2012 Jan [Citado 2016 Jan 20]; 38(2): 286-291. Disponible en: http://www.scielos p.org/scielo.php?script=sci_arttext\&pid= S0864-34662012000200012\&Ing=en. http://dx.doi.org/10.1590/S0864-346620 12000200012.

18. Jones, K. B. The staff of Asclepius: a new perspective on the symbol of medicine. WMJ MADISON [Internet]. 2008 [citado en 2016 Jan 20]; 107(3): 115. Disponible en: https://www.wisconsinmedicalsociety. org/_WMS/publications/wmj/pdf/107/3/115. pdf.

19. Stenn, F. The symbol of medicine. Quarterly Bulletin of the Northwestern University Medical School [Internet]. 1958 [citado en 2016 Jan 20]; 32(1): 74-87. Disponible en: http://www.ncbi.nlm.nih.gov/pmc/articles/ PMC3803633/.

20. Prates Paulo R. Do bastão de Esculápio ao caduceu de Mercúrio. Arq. Bras. Cardiol. [Internet]. 2002 Oct [cited 2016 June 12] ; 79 (4): 434-436. Available from: http:/ www.scielo.br/scielo.php?script=sci_arttext\&pid=S0066-782X2002001300014\&Ing= en.http://dx.doi.org/10.1590/S0066-782X 2002001300014.

21. Montoya Alvares, Juan. Historia del símbolo de la medicina. Revista Médica Hondureña [Internet]. 1947 [citado en 2016 Jan 20]; 17 (3):194. Disponible en: http://www.bvs. hn/RMH/pdf/1947/pdf/A17-3-1947-2.pdf. 
22. F. S. FAN. The symbol of medicine in ancient China. SAMJ [Internet]. 1992 Oct [citado en 2016 Jan 20]; 82: 273-274. Disponible en: http://www.ncbi.nl m.nih.gov/ pubmed/1411829.

23. Santa Biblia. Versión Reina-Valera 1960 [en línea]. Sociedades Bíblicas Unidas. 1988. Génesis 3:1-4. [Citado 2016 Mar 05] Disponible en: https:// www.biblega teway.$\mathrm{com} /$ passage/?search=Genesis+3\%3A1-4\&ver sion=R VR1960.

24. Santa Biblia. Versión Reina-Valera 1960 [en linea]. Sociedades Bíblicas Unidas. 1988. Números 21:4-9. [Citado 2016 Mar 05]. Disponible en: https://www. biblegateway. com/passage/?search $=\mathrm{N} \% \mathrm{C} 3 \% \mathrm{BA}$ meros $+21 \% 3 A 4-9 \&$ version= RVR1960.

25. Santa Biblia. Versión Reina-Valera 1960 [en línea]. Sociedades Bíblicas Unidas. 1988. Éxodo 7:9-12. [Citado 2016 Mar 05] Disponible en: https://www.biblega teway. $\mathrm{com} /$ passage/?search=Ex+7\%3A9-12\&ver sion =RVR1960.
26. Pérez Vázquez IA, Sánchez Lera RM. EI bastón de Esculapio: su historia. Humanidades Médicas [Internet]. 2014 [citado en 2016 Jan 20]; 14(1):220-237. Disponible en: http://scielo.sld.cu/scielo.php?script=sci_ar ttext\&pid= S1727-81202014000100014.

27. Currie, A. True symbol of medicine. Canadian Family Physician [Internet]. 2000 [citado en 2016 Jan 20]; 46: 274. Disponible en: http://www.ncbi.nlm.nih.gov/pmc/articles/ PMC1987683/.

28. Vidal M, Borroto ER, Delgado G. El caduceo de la Medicina, símbolo distintivo y emblema oficial de la Salud Pública cubana [internet] [Citado 2016 June 12]; Educ Med Super 2004; 18(2). Disponible en: http:// bvs.sld.cu/revistas/ems/vol18_2_04/ems 06204.htm.

29. Caiman, Kenneth. The arrow or the Caduceus as the symbol of the doctor. The Lancet [Internet]. 2003 Jul [Citado en 2016 Jan 20]; 362 (9377): 84. Disponible en: http://www.thelancet.com/jour nals/la cet/ar ticle/PIIS0140-6736(03)13 833-0/abstract. 